\title{
A PhD state of mind
}

\author{
Recent surveys have linked academia and PhD studies to a risk of experiencing mental health issues. Despite \\ the lack of extensive data, the negative impact of the stresses of lab life should not be underestimated, and PhD \\ students and research trainees should be supported.
}

t is an uncomfortable fact of lab life
that a student in pursuit of a doctorate
is likely to experience the PhD blues
at some point in his or her training. The
unsuccessful project, the experiment that
is resistant to troubleshooting, the pressure
to succeed, the uncertain career path, can
all take their toll on the graduate student
psyche. Most PhD graduates have stories
of high stress or dejection, and of long
evenings and weekends in the lab trying to
get an experiment to work, to complete a
manuscript for submission or to finish their
thesis. The anecdotal evidence of a high-
pressure environment is supported by recent
reports. Nature's 2017 survey on the lives and
career aspirations of $~ 5,700$ PhD students
worldwide (http://go.nature.com/2qWSyfX)
reported that although roughly three quarters of respondents were satisfied with their PhD programmes overall, more than a quarter saw mental health as an area of concern, with $12 \%$ having sought help for anxiety or depression caused by their $\mathrm{PhD}$ studies. A 2017 study querying 3,596 PhD students at universities in Flanders, Belgium (http://doi.org/b4wm), reported that a third of respondents were at risk of having or developing a common psychiatric disorder, such as depression. A 2014 study at the University of California, Berkeley (http:// go.nature.com/2HwlL4v) found high levels of depression among 790 postgraduate students with $47 \%$ of $\mathrm{PhD}$ students meeting the criteria to be classified as depressed.

On reviewing the literature on mental health in research environments, a 2017 report by the Royal Society and the Wellcome Trust in the UK (http://go.nature. $\mathrm{com} / 2 \mathrm{p} 8 \mathrm{fq} 8 \mathrm{R})$, noted that the evidence is too limited to permit robust conclusions about the mental health status and needs of researchers. Nevertheless, the potential mental impact of pursuing a $\mathrm{PhD}$ should not be disregarded. Even though $\mathrm{PhD}$ programmes vary widely, the life of the average new PhD student undergoes major shifts in terms of structure, responsibilities and expectations of success compared to the demands of an undergraduate degree. Classrooms and exam halls are swapped for the bustling and sometimes pressure-cooker environment of a lab. A single supervisor and small thesis committee replace the numerous university lecturers and tutors, and the large population of undergraduate peers is reduced to a smaller group of labmates of different career stages and ambitions. Curricula common to all students give way to specialised projects driven by the individual, and the exams that punctuate the university calendar and offer a finite measure of success are replaced by the larger, more abstract goals of making new scientific discoveries worthy of publication and a doctorate. Even when starting a $\mathrm{PhD}$ on the back of a Masters degree, the unique rhythms of lab life can take some getting used to. The calm periods of projects quietly chugging along can quickly give way to phases of frenzied work, and the highs of an exciting result are often followed by the frustration and low spirits caused by a failed experiment.

At the core of these pressures is the nature of the scientific endeavour itself. Getting a project off the ground and seeing it to completion requires hard work and dedication, analytical but also creative thinking, self-motivation and resilience. Researchers have to harden themselves to failure, but the pressure to obtain results and succeed, often while facing competition, can be stressful even for the most strongwilled scientist. The high stakes and daily hardships of research can seed feelings of isolation and personal failure during the long years of a $\mathrm{PhD}$. The time demands of lengthy and complex experiments can also lead to unpredictable working hours, skewing work-life balance and creating a sense of loss of control. The ability to conduct multiple projects in parallel might overload those with less honed organisational skills, and can lead to burnout when plans become overambitious. Pressures of time and funding can also take a toll, especially in countries with short $\mathrm{PhD}$ programmes. Fear of failure and the repercussions this might have for long-term professional development and job security can be the source of anxiety.Uncertainty about the future can loom large for trainees, especially at times of limited science budgets that make the more traditional path of academia a less viable option.

There is no one-size-fits-all solution to addressing the complex issue of mental health and wellbeing in the workplace. Thus, it is essential for $\mathrm{PhD}$ programmes to provide an environment able to recognise signs of mental distress and respond with guidance and appropriate resources. Good mentoring (http://doi.org/cmb9) and an open and productive relationship with the $\mathrm{PhD}$ advisor can offset many of the daily pressures faced by students. Cultivating a lab culture that aims for work-life balance and productivity, rather than burnout and presenteeism, is key. Discouraging dysfunctional lab situations such as internal competition and isolationism, promoting communication and cooperation, encouraging senior lab members to take mentoring roles, and supporting social, team-building interactions can make the lab a thriving, inviting environment. Inspiring a sense of accomplishment in smaller achievements a well-performed experiment, a successful presentation, a productive collaboration can increase the day-to-day satisfaction in one's work. At the university and institute level, fostering closer ties within the $\mathrm{PhD}$ student body and organising regular meetings with thesis committee members can offer crucial support outside the lab, including much needed reality-checks on progress and perceived failures. Ensuring that advisers, thesis committees and $\mathrm{PhD}$ programme administrators offer guidance about career paths can also counteract a large source of stress. It is essential to note that $\mathrm{PhD}$ administrators and supervisors should not be called to address mental health problems experienced by students. Rather, they should offer support by listening and directing students to suitable resources for obtaining further help, including by qualified professionals as needed. Indeed, many universities and institutes offer counselling services staffed by qualified, trained counsellors.

$\mathrm{PhD}$ programmes, research departments and group leaders should aim to nurture the passion, creativity and drive of budding scientists. Recognising the negative impact of daily lab stresses on the individual, and cultivating a culture of openness, are key steps towards establishing appropriate support systems to help students overcome the pressures of the research world.

Published online: 28 March 2018 https://doi.org/10.1038/s41556-018-0085-4 